\title{
Clinical Effect of Magnetic Resonance Imaging and 64-slice Spiral CT in the Diagnosis of Ischemic Heart Disease Patients
}

\author{
Jiamei Wang ${ }^{1}$, Xianling Zheng ${ }^{2}$, Hongfeng Zhang ${ }^{3}$, Junjuan $\mathrm{Qi}^{{ }^{\star}}$, Shifeng Xiang ${ }^{{ }^{\star}}$ \\ ${ }^{1}$ Department of Imaging, Handan Central Hospital, Handan 056001, Hebei Province, China \\ ${ }^{2}$ Department of Endocrinology, Handan Central Hospital, Handan 056001, Hebei Province, China \\ ${ }^{3}$ Scientific Research Department, Handan Central Hospital, Handan 056001, Hebei Province, China \\ ${ }^{4}$ Hospital Office, Handan Central Hospital, Handan 056001, Hebei Province, China
}

\begin{abstract}
[Abstract] Objective: To explore the clinical methods and clinical effects of applying magnetic resonance imaging (MRI) and 64-slice spiral computed tomography (CT) in the diagnosis of patients with ischemic heart disease. Methods: 100 patients with ischemic heart disease were selected as the research objects. Selecting the patients from May 2020 to May 2021 as a sample, the patients were divided into two groups, and different diagnostic methods were used to compare the clinical diagnosis effects. Results: In terms of the diagnostic accuracy of the two groups of patients, the maximum value was $92.00 \%$ (experimental group) and the minimum value was $80.00 \%$ (control group). There was a big difference in data between the two groups, $P<0.05$, which was statistically significant. The patient's (experimental group) diagnosis accuracy rate is higher. Conclusion: In the process of research work for patients with ischemic heart disease, it is particularly important to diagnose the patients. The combined application of and 64-slice spiral CT can improve the clinical diagnosis efficiency and achieve significant results.
\end{abstract}

Key words: Magnetic resonance imaging; 64-slice spiral CT; Ischemic heart disease; Patient diagnosis

Publication date: May, 2021; Publication online: 31 May, 2021

*Corresponding author: Junjuan Qi, qijunjuan899@sina.cn; Shifeng Xiang, xsf51460855@sina.com

\section{Introduction}

Clinical trials for ischemic heart disease are very common, with coronary atherosclerotic heart disease being the main type. In conjunction with China's current development, various economic constructions have improved, and people's quality of life have improved to a certain extent which led to changes in people's living habits and dietary structure. However, China is gradually developing in the direction of aging, thus increasing the incidence of diabetes in patients. Since diabetes is the main factor leading to the occurrence of ischemic heart disease, the clinic has gradually strengthened the importance of patients ${ }^{[1]}$. Ischemic heart disease poses a greater threat to the patient's health, whereby clinically the patient's condition can only be controlled but not cured. Therefore, it is particularly important to clarify the patient's condition is in the early stage before starting the trial, and it is important to avoid the deterioration of the patient's condition. On this basis, this study combines MRI and 64-slice spiral CT diagnostic methods to explore its impact on patients, and the analysis is as follows.

\section{Materials and methods}

\subsection{General data analysis}

The research work was carried out from May 2020 to May 2021. Selecting 100 patients with ischemic heart disease as a sample, the patients were diagnosed and different diagnostic methods were implemented. Therefore, the patients were divided into two groups, where each group has 50 cases. In 
the experimental group, male: 25 cases, female: 25 cases, age range $52-87$ years, average $(66.55 \pm 3.94)$ years old. In the control group, male: 26 cases, female: 24 cases, age range 53-88 years old, mean $(67.04 \pm 3.82)$ years old. The data of the patients in this study were compared under statistical methods, expressed as $P>0.05$, the data difference is small and unimportant, and the diagnostic effect can be used as the only variable in this study.

\subsection{Research methods}

The patients in the control group used pure MRI diagnosis. In the specific implementation process, the 3T MRI scanner is mainly used. This instrument is mainly produced by Twin Speed GE. It is adjusted to the specified magnification mode and various parameters were performed. Set the gradient field intensity as controlled at $40 \mathrm{mT} / \mathrm{m}$, the maximum switching rate is $150 \mathrm{~T} / \mathrm{m} / \mathrm{s}$, and the cardiac phased array coil is applied. During the inspection process, the extracellular contrast agent given to the patient were mainly Gd-DTPA produced by Schering, Germany, and its application dose was controlled at $0.1 \mathrm{mmol} / \mathrm{L}$. After the first application, wait till the perfusion is completed before scanning. After 5 minutes, perform a layer-by-layer delayed scan. The main inspection sites include the short axis, four-chamber heart position, and the long-axis two-chamber heart position. The data is set, the repetition time is $3.4 \mathrm{~ms}$, and the echo time is $1.4 \mathrm{~ms}$, the reversal angle is 20 degrees, the reversal time is $200 \mathrm{~ms}$, the corresponding acquisition matrix is $256 \times 192$, and the scanning field of view is controlled at $36 \times 27 \mathrm{~cm}$, and the layer thickness is controlled at $8-10 \mathrm{~mm}$.

The patients in the experimental group used a combination of MRI scanning and 64-slice spiral CT scanning. The MRI scanning method and equipment used are the same as those of the control group. The 64-slice spiral CT scan requires: before the examination, give the patient an iodine dose and monitor the patient's heart rate. If the patient's heart rate is below 70 beats $/ \mathrm{min}$, it indicates that the patient can accept the scan. Otherwise, the examination cannot be performed. Therefore, the patient needs to be given metoprolol tablets for intervention. Upon achieving the standard of the patient's heart rate level, then the patient can be scanned. When scanning, the choice of contrast agent is mainly Onipak, which is injected at a speed of $5 \mathrm{ml} / \mathrm{s}$. When the signal in the ascending aorta is observed to reach $100 \mathrm{HU}$, the patient can be scanned. Nursing staff needs to guide the patient to cooperate with the examination, tell the patient to hold their breath, the time is $8-9 \mathrm{~s}$, and record the patient's ECG changes. After the scan is completed, it is necessary to analyze the data obtained from the scan, take cross-sectional reconstruction, and process the reconstructed image. For the cross-sectional image, the volume reproduction is enhanced, and the largest area projection is processed, and the left main trunk, left anterior descending branch, circumflex branch, as well as right coronary artery is reconstructed in $2 \mathrm{D}$ and $3 \mathrm{D}$, only then the image is analyzed.

\subsection{Observation index}

The two groups of images were analyzed to determine the patient's condition, and the number of people diagnosed under the two inspection methods was statistically compared.

\subsection{Statistical methods}

The statistical software SPSS20.0 was used as a tool to perform statistical analysis on the data presented in this study. It verified the comparison results of the measurement data $(\mathrm{x} \pm \mathrm{s})$ with the $\mathrm{t}$ value, and compare the count data $(\mathrm{n}, \%)$. The results were verified by the $\mathrm{x} 2$ value. When the results showed $P<0.05$, it indicated that the differences between the groups had statistical analysis value.

\section{Results}

In terms of the diagnostic accuracy of the two groups of patients, the maximum is $92.00 \%$ (experimental group) and the minimum is $80.00 \%$ (control group). There is a big difference in data between the two groups, $P<0.05$, and the diagnostic accuracy of the experimental group is higher.

Table 1. Comparison of the diagnostic accuracy of the two groups of patients $[\mathrm{n}(\%)]$

\begin{tabular}{ccc}
\hline Group & $\begin{array}{c}\text { The number of } \\
\text { confirmed (n) }\end{array}$ & $\begin{array}{c}\text { Diagnostic } \\
\text { accuracy (\%) }\end{array}$ \\
\hline Test group $(\mathrm{n}=50)$ & 46 & $92.00 \%$ \\
$\begin{array}{c}\text { Control group } \\
(\mathrm{n}=50)\end{array}$ & 40 & $80.00 \%$ \\
$\mathrm{X}^{2}$ & & \\
$P$ & & \\
& & $<.445$ \\
\hline
\end{tabular}

\section{Discussion}


With the continuous advancement of China's economic construction, the incidence of cardiovascular and cerebrovascular diseases are gradually increasing. Among them, ischemic heart disease is a majority, which poses a greater threat to the health of patients. It is important to treat patients clinically and improve the accuracy of diagnosis ${ }^{[2]}$. With the continuous advancement of China's medical development, MRI technology has been improved, and cardiac magnetic resonance imaging (CMR) technology has grown in a short period of time, also has been widely used in clinical trials. It has become the main standard for evaluating the structure and function of the heart. Moreover, patients with ischemic heart disease have a positive effect ${ }^{[3]}$ with CMR technology. However, in actual development, CMR technology has relatively high operational complexity, whereby it requires high performance of machinery and equipment, also requires high work experience and technical foundation for operators. From the current clinical development, this technology can only be used in a few diagnostic treatments which makes it is difficult to be promoted and popularized for hospital use ${ }^{[4]}$. On this basis, if this study conducted its research on CMR technology, the procedure would be as, selected patients with ischemic heart disease from our hospital as a sample, used 3T MRI scanner, then expanded MRI routine sequence using multi-b value Diffusion-weighted imaging (DWI) sequence, and after passing the perfusion sequence, conducted a delayed enhancement sequence examination with the intravoxel incoherent motion (IVIM) technology which can achieve the myocardial examination of patients with old myocardial infarction, determine the patient's myocardial condition, and obtain the infarcted myocardial $\mathrm{D}$ value, $\mathrm{D}^{*}$ value and f-value parameter values. These values are paired with the assessment of the degree of myocardial infarction fibrosis is of positive significance, which can assist doctors in determining the patient's myocardial blood perfusion and water molecule diffusion to achieve a better assessment on the patient's condition ${ }^{[5]}$. When compared to the enhanced examination technique, this technique is more effective in diagnosing patients and allows for a better observation of the patient's myocardial infarction position, infarction percentage and degree of transmural, cardiac function, and viable myocardium after infarction. In order to provide patients targeted treatment according to their actual conditions $^{[6]}$

In this study, after the combined application of 3T MRI and 64-slice spiral CT, the results showed that the diagnostic efficiency of the experimental group was significantly higher than that of the control group, and the data between the groups were significantly different, when expressed as $\mathrm{P}<0.05$, which was statistically significant. It shows that the combined detection of the two methods has a significant effect on patients.

In summary, it is particularly important to diagnose patients with ischemic heart disease. In this process, the combined application of MRI and 64-slice spiral CT can improve the clinical diagnosis efficiency as the effect is significant, and simultaneously it can be popularized.

\section{Disclosure statement}

The author declares no conflict of interest.

\section{References}

[1] Xiang, SF., Zhang, XQ., Yang, SJ., et.al (2019). Intravoxel Incoherent Motion Magnetic Resonance Imaging with Integrated Slice-specifc Shimming for old myocardial infarction: A Pilot Study. Scientific Reports,9.

[2] Xiang, SF., Zhang, XQ., Yang, SJ., et.al (2018). STROBE-A Preliminary Investigation of IVIM-DWI in Cardiac Imaging. Medicine,97:36(e11902).

[3] Xiang, SF., Zhang, XQ., Yang, SJ., et.al (2018). Application of Diffusion-Weighted Magnetic Resonance Imaging of Incoherent Motion within Voxel in Cardiac Imaging[J]. Shandong Medicine,58(17): 46-48.

[4] Xiang, SF., Zhang, XQ., Yang, SJ., et.al (2018). The Value of Cardiac Magnetic Resonance Imaging in the Differential Diagnosis of Left Ventricular Hypertrophy [J]. Journal of Medical Imaging,28(6): 920-922.

[5] Xiang, SF., Zhang, XQ., Yang, SJ., et.al (2017). Analysis of MRI Manifestations and Electrocardiogram Characteristics of Patients with Apical Hypertrophic Cardiomyopathy[J]. Journal of Integrated Chinese and Western Medicine Cardio-Cerebrovascular Disease, 15 (10): 1280-1282.

[6] Xiang, SF., Song, LH., Yang, SJ., et.al (2014). MRI Diagnosis of Left Ventricular Myocardial Insufficiency [J]. Journal of Medical Imaging,24 (1): 56-58. 